\title{
Communication
}

\section{Presence of Rickettsia Species in Ticks Collected from Companion Animals in Northeastern Georgia, United States}

\author{
Hannah Stanley and DeLacy V. L. Rhodes* \\ Department of Mathematics and Natural Sciences, Berry College, Mount Berry, GA 30149, USA; \\ Hannah.Stanley@vikings.berry.edu \\ * Correspondence: drhodes@berry.edu
}

Citation: Stanley, H.; Rhodes, D.V.L. Presence of Rickettsia Species in Ticks Collected from Companion Animals in Northeastern Georgia, United States. Vet. Sci. 2021, 8, 37. https:// doi.org/10.3390/vetsci8030037

Academic Editors: Stephen Kenneth Wikel and Antonio Humberto Hamad Minervino

Received: 31 January 2021

Accepted: 24 February 2021

Published: 26 February 2021

Publisher's Note: MDPI stays neutral with regard to jurisdictional claims in published maps and institutional affiliations.

Copyright: (c) 2021 by the authors. Licensee MDPI, Basel, Switzerland. This article is an open access article distributed under the terms and conditions of the Creative Commons Attribution (CC BY) license (https:// creativecommons.org/licenses/by/ $4.0 /)$.

\begin{abstract}
Tick-borne diseases are a major threat to both humans and their pets; therefore, it is important to evaluate the prevalence of pathogens carried by ticks on companion animals. In this study, attached and unattached Ixodid ticks were removed from companion animals by a veterinary practice in Hall County, Georgia. DNA was extracted from unengorged adult ticks and each was screened for the presence of Rickettsia spp. by polymerase chain reaction (PCR) and sequenced to determine the species present. Two hundred and four adult hard-bodied ticks were identified to species and Rickettsia spp. were found in 19.6\% $(n=38)$ of the 194 analyzed DNA extracts. Rickettsia montanensis was found in Dermacentor variablis $(14.7 \% ; n=25)$, Amblyomma maculatum $(33.3 \% ; n=2)$, and Rhipicephalus sanguineus s.l. ticks $(25 \% ; n=4)$. One Amblyomma americanum tick contained Rickettsia amblyommatis, while Rickettsia felis was found in one Dermacentor variablis tick, serving as the first report of Rickettsia felis in a tick in this region and within this tick vector. This study suggests that there is a risk of companion animals contracting a species of Rickettsia from a tick bite in northeastern Georgia, indicating a need for more investigation and highlighting the importance of tick prevention on pets.
\end{abstract}

Keywords: companion animals; Rickettsia; tick-borne diseases; tick prevention

\section{Introduction}

Tick-borne diseases are among the most prevalent vector-borne diseases in the United States. According to the Centers for Disease Control and Prevention, incidence rates for the most common tick-borne diseases in the United States, such as Lyme disease and Rocky Mountain spotted fever, have been steadily increasing over time. [1-3]. Although improved diagnostics and public awareness have increased understanding of the incidence and distribution of these tick-borne diseases, these infections still remain underrepresented due to lack of reporting and misdiagnosis. [4,5]. As cases of these tick-borne diseases continue to rise, the study of their causative agents is an essential step toward understanding their prevalence and decreasing their incidence.

Of the seven species of Ixodid ticks that bite and transmit disease to humans in the United States, five are found in Georgia, including Dermacentor variabilis, Ixodes scapularis, Rhipicephalus sanguineus (sensu lato), Amblyomma maculatum, and Amblyomma americanum. Each of these tick species is known to be the vector for different tick-borne infectious agents, including several different species of Rickettsia [6-10]. Rickettsia are small, Gram-negative intracellular bacteria that are broken into two traditional groups-the typhus group and the spotted fever group [11]. Members of the spotted fever group (SFG) Rickettsia cause several different illnesses such as Rickettsia parkeri rickettsiosis, caused by R. parkeri, and Rocky Mountain spotted fever (RMSF), caused by $R$. rickettsii $[12,13]$. An additional species of SFG Rickettsia more commonly associated with fleas, $R$. felis, has recently been found to be an important human pathogen in sub-Saharan Africa, where it is responsible for flea-borne spotted fever [14,15]. In addition to the species of Rickettsia that are associated with infectious disease, there are many species that are considered nonpathogenic. These 
various rickettsial endosymbionts, such as $R$. bellii, are associated with many different species of ticks and are not currently known to cause human illness [16].

Humans typically contract tick-borne pathogens through the bites of questing ticks that are acquired through outdoor activities or through close contact with animals transiently harboring ticks that are carrying pathogens. Companion animals can act as reservoirs for some tick-borne pathogens and are risk factors for all human age groups due to the introduction of ticks into people's homes [17-19]. In addition to acting as hosts for infected ticks, domestic cats and dogs are susceptible to infection from certain tick-borne pathogens as well [20]. It is, therefore, important to evaluate the prevalence of pathogens carried by ticks on companion animals. In this study, ticks removed from companion animals by a veterinary practice in northeastern Georgia were screened individually for rickettsial species. By assessing the pathogen infection rates of ticks collected from companion animals, insight can be gained into the risk of humans and animals contracting these illnesses as well as into the risk companion animals may pose to their owners through the exposure of ticks that harbor these pathogens.

\section{Materials and Methods}

\subsection{Tick Collection and Identification}

Ticks used in this study were removed from companion animals between April and October of 2016 by a veterinarian office in Hall County, Georgia and stored in isopropyl alcohol. Attached and unattached ticks were removed during routine visits with 1-3 ticks removed per animal. For this study, only unengorged adult Ixodid ticks were analyzed. Ticks were identified visually using a dissecting microscope and a published identification guide [21].

\subsection{DNA Extraction}

DNA from all ticks was extracted using a commercially available DNA purification kit (GeneJET Genomic DNA Purification Kit, Thermo Fisher Scientific, Inc., Waltham, MA, USA). Instructions for extracting DNA from Gram-negative bacteria provided by the manufacturer were followed with minor alterations. An overnight digestion step was added before the manufacturer's protocol steps were begun. Each tick was quartered and digested overnight with Proteinase $\mathrm{K}$ and included lysis buffer in a $56{ }^{\circ} \mathrm{C}$ water bath Following $\sim 16 \mathrm{~h}$ of digestion, the samples were thoroughly vortexed and centrifuged at $8000 \times g$ to pellet the tick exoskeleton. The supernatant was then transferred to a new tube and the manufacturer's protocol was followed. Extracted DNA was stored in a refrigerator until screening and then frozen after.

\subsection{DNA Amplification}

DNA samples were screened individually by polymerase chain reaction (PCR) for members of the genus Rickettsia. Additionally, a control reaction was performed with each sample in which the tick 16S rRNA gene was amplified. The purpose of this control was to demonstrate that DNA was successfully extracted from each sample. The primers for this control were designed for this study (Tick $16 \mathrm{~s}$ Fwd: TTG CTG TGG TAT TTT GAC TAT ACA AAG GTA; Tick 16s Rev: CCG GTC TGA ACT CAG ATC). A commercially available Taq polymerase master mix (GoTaq ${ }^{\circledR}$ Green Master Mix, Promega Biosciences, LLC, Madison, WI, USA) and its accompanying protocol were used for all screening reactions. Nested PCR was used for Rickettsia spp. detection by amplification of the ompA gene as previously described [22]. For use as a positive control, omp $A$ was amplified from $R$. parkeri genomic DNA and cloned into $\mathrm{PCR} 2.1$ following the manufacturer's instructions for TOPO cloning (Invitrogen, Carelsbad, CA). A negative control using water in the place of DNA was also included in all primary and secondary reactions. PCR products were run on $1 \%$ agarose gels (Bio-Rad, Hercules, CA, USA) and were stained with $1 \%$ ethidium bromide (Sigma-Aldrich, Saint Louis, MO, USA). 


\subsection{DNA Sequencing and Analysis}

DNA sequencing was used to determine the species of all positive Rickettsia spp. samples [22]. The samples that yielded a positive ompA amplicon in the secondary reaction were purified from agarose gels using a gel extraction kit (QIAquick Gel Extraction Kit, Qiagen, Inc., Hilden, Germany) following the manufacturer's instructions. Samples were sequenced by GenScript, LLC. (Piscataway, NJ, USA) on a fee for hire basis. Positive ompA sequences were analyzed using ClustalX for alignment [23].

The species of Rickettsia found in each tick was determined by BLASTn searching of the NCBI database and phylogenetic analysis using maximum likelihood methods [24]. For each sample, 544 base pairs of sequence were aligned and analyzed. Consensus sequences for comparison were acquired from GenBank and are listed in Table S1 along with accession numbers. Table $\mathrm{S} 2$ shows the percent similarities of all sequences analyzed in this study as determined by NCBI BLASTn searching, along with the sex of each individual tick that tested positive for Rickettsia. All sequences generated in this study are included in Data S3 and the phylogenetic tree is included as Figure S1.

\section{Results}

\subsection{Tick Assemblages}

A total of 204 adult unengorged ticks were collected for this study. Of these ticks, four species were found. A total of 180 were identified as D. variabilis, 16 as Rhipicephalus sanguineus s.l., six as A. maculatum, and two as A. americanum (Table 1). Of the collected ticks, 114 were male while 80 were female. No I. scapularis ticks were collected in this study, although this species of tick is present in Georgia.

Table 1. Total numbers of tick species and associated Rickettsia species.

\begin{tabular}{|c|c|c|c|c|c|c|}
\hline \multirow{6}{*}{ 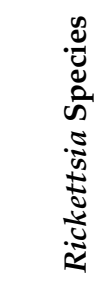 } & & \multicolumn{4}{|c|}{ Tick Species } & \multirow[b]{2}{*}{$\begin{array}{l}\text { Total Per Species } \\
\text { (\% Positive) }\end{array}$} \\
\hline & & $\begin{array}{l}\text { Dermacentor } \\
\text { variablis }\end{array}$ & $\begin{array}{l}\text { Amblyomma } \\
\text { americanum }\end{array}$ & $\begin{array}{l}\text { Amblyomma } \\
\text { maculatum }\end{array}$ & $\begin{array}{l}\text { Rhipicephalus } \\
\text { sanguineus s.l. }\end{array}$ & \\
\hline & R. montanensis & $25(14.7 \%)$ & $0(0 \%)$ & $2(33.3 \%)$ & $4(25 \%)$ & $31(34.1 \%)$ \\
\hline & R. felis & $1(0.6 \%)$ & $0(0 \%)$ & $0(0 \%)$ & $0(0 \%)$ & $1(1.01 \%)$ \\
\hline & R. amblyommatis & $0(0 \%)$ & $1(50 \%)$ & $0(0 \%)$ & $0(0 \%)$ & $1(1.01 \%)$ \\
\hline & Unknown & $3(1.8 \%)$ & $0(0 \%)$ & $2(33.3 \%)$ & $0(0 \%)$ & $5(5.5 \%)$ \\
\hline \multicolumn{2}{|c|}{$\begin{array}{l}\text { Total Rickettsia Positive/Total } \\
\text { Tick Species (\% positive) }\end{array}$} & $29 / 170(17 \%)$ & $1 / 2(50 \%)$ & $4 / 6(66.7 \%)$ & $4 / 16(25 \%)$ & $38 / 194(19.6 \%)$ \\
\hline
\end{tabular}

\subsection{PCR Screening and Species Identification}

A total of 10 ticks tested negative with the tick $16 \mathrm{~S}$ primer set, suggesting unsuccessful DNA extraction, and were removed from the analysis. This brought the total number of ticks tested for pathogen presence to 194. Of the 194 ticks tested, Rickettsia spp. were detected in $19.6 \%$ of all the ticks analyzed $(n=38)$. By BLASTn analysis and phylogenetic analysis, 31 of the positive samples were identified to be $R$. montanensis, 1 was $R$. amblyommatis, and 1 was $R$. felis. The one $R$. amblyommatis sequence was identified in an $A$. americanum tick while the one $R$. felis sequence was identified in a $D$. variabilis tick. The sequences that aligned with $R$. montanensis were found in $25 \mathrm{D}$. variabilis ticks $(14.7 \%)$, 2 A. maculatum ticks (33.3\%), and 4 Rhipicephalus sanguineus s.l. ticks (25\%) (Table 1 ). Percentages in columns represent the percentage of each tick species positive for each Rickettsia species. Phylogenetic analysis supported BLAST sequence identification with high bootstrap values for each species identified (Figure S4). Five samples (Am002, Am003, Dv147, Dv162, and Dv127) tested positive for the presence of Rickettsia DNA by PCR but did not align with any tested Rickettsia species within the $98 \%$ sequence identity threshold values of this study. Therefore, these samples were removed from our analysis. Due to the sensitivity of nested PCR and the use of R. parkeri DNA as a positive control, this species of Rickettsia was omitted from this analysis. 


\section{Discussion}

The most commonly collected ticks identified in this study were D. variabilis ticks, comprising $88 \%$ of the total ticks collected. Despite being the most common tick species found in Georgia, there were only two A. americanum ticks in the pool $[5,9]$. Additionally, it is interesting that although Rhipicephalus sanguineus s.l. prefers domestic dogs as hosts, only a small number of this species of tick $(n=16)$ was found on companion animals as part of this study [25,26]. As mentioned above, no I. scapularis were collected and analyzed in this study. This is most likely due to the fact that tick collections took place outside of the normal questing period for adult I. scapularis ticks in the southeastern United States [27]. A larger number of male ticks were analyzed than female ticks in this study. This is unsurprising due to the fact that only unengorged ticks were analyzed.

Approximately $19.6 \%(n=38)$ of the ticks in this study harbored a species of Rickettsia with three different species of Rickettsia identified. Two known rickettsial species were found in one tick each-R. felis and R. amblyommatis (Table 1). The discovery of R. felis is an important and surprising finding. While the occurrence of infections in the United States is low, R. felis is a common cause of flea-borne fever worldwide [15,28]. This species was once known only as a flea-borne species, but it has since been identified that over 40 species of fleas, ticks, mites, and mosquitoes can harbor the pathogen [14,15]. Although the full vector competency of $R$. felis is still under investigation, several species of ticks have been found to harbor R. felis including Haemophysalis suldata, Haemophysalis flava, Haemophysalis kitaokai, Ixodes ovata, and Rhiphicephalis sanguinius [29-31]. Fewer studies have investigated R. felis in ticks in the United States, focusing instead on the prevalence of this pathogen in fleas and vertebrate hosts, though one US-based study did identify $R$. felis associated with A. maculatum ticks obtained from humans in the southern United States [32]. While cofeeding cannot be excluded in this study as a potential cause of the positive identification of $R$. felis in a tick, studies that have identified R. felis with environmentally or humancaptured ticks along with the use of a tick cell culture model suggest that ticks may be able to harbor this pathogen [31-33]. This study identified one D. variabilis tick to be positive with $R$. felis, representing the first finding of $R$. felis in a tick in Georgia and the first finding of $R$. felis within a Dermacentor tick.

This study identified one A. americanum tick containing R. amblyommatis, a rickettsial species that is still being investigated to understand its full pathogenic potential. The investigation of humans seropositive for various species of SFG Rickettsia has suggested that R. amblyomatis (formerly known as Candidatus Rickettsia amblyomii) may be responsible for less severe cases of RMSF in the southeastern United States [34-37]. Another study looking into natural infection of dogs by ticks showed that dogs produced high antibody titers against $R$. amblyommatis, suggesting that dogs are able to be infected with this species [38]. As rickettsioses can be difficult to diagnose in canines, it is reasonable that this species may be a cause of disease in canine that is often overlooked [39].

The most prevalent species of Rickettsia identified in this study was R. montanensis, found in $34.1 \%(n=31)$ of screened ticks. The ability of this rickettsial species to infect dogs has been investigated and it is not associated with any clinical symptoms in canines when infected both experimentally and naturally $[38,40]$. Additionally, it has been found that dogs mount a strong antibody response against $R$. montanensis and, though antibody cross-reactivity is common amongst the SFG rickettsiae, this antibody response does not provide protection against infection with some pathogenic species of Rickettsia [38-40]. In this study, twenty-five $D$. variabilis ticks (14.7\%), two A. maculatum ticks $(33.3 \%)$, and four Rhipicephalus sanguineus ticks $(25 \%)$ were found to harbor $R$. montanensis (Table 1 ). Though nonpathogenic in dogs, there has been a single case of an afebrile rash illness in a six-year-old girl from Georgia who was bitten by a $D$. variabilis tick carrying $R$. montanensis, indicating that this organism may occasionally be able to cause disease and underscoring the importance of the use of tick preventative treatment on household pets [41]. Additionally, it is speculated that colonization by R. montanensis in the tick can outcompete R. rickettsii [7]. As this is a disease known to occur in northern Georgia, the prevalence of 
$R$. montanensis in ticks found in this study may have contributed to the absence of $R$. rickettsii in our samples [7].

This study is small in scope and contains limitations that are important to note. For one, the ticks collected in this study were donated by a veterinary clinic and no data were collected that associate the individual ticks with the animal(s) from whom they were collected. Therefore, information concerning the species of the associated animal hosts (i.e., cats versus dogs) and the number of ticks collected from each individual animal is unavailable. Additionally, one gene was assessed to determine Rickettsia species, though it was analyzed through multiple methods. Greater information into the individual ticks and the analysis of an additional gene for the determination of Rickettsia species would have strengthened our results.

\section{Conclusions}

Ticks collected from companion animals and veterinary offices provide a unique perspective into the human and animal risk for exposure to ticks and tick-borne diseases. Studying ticks collected in this manner provides important insight into the prevalence of tick-borne pathogens while also underscoring the need for regular tick prevention and removal from companion animals. This study has shown that pets can bring ticks potentially harboring pathogens into homes, increasing the risk of tick-borne diseases to pet owners. Almost a quarter of all the ticks analyzed in this study (19.6\%) contained some species of Rickettsia (Table 1). This study has also identified a known pathogenic species, $R$. felis, to be present in D. variablis ticks in Georgia. While greater information into the history of the ticks and animals utilized in this study and additional genetic analysis could provide important information to the analysis of this study, this work has regional significance and provides data that are important in helping veterinarians impress the need for regular tick prevention on pets.

Supplementary Materials: The following are available online at https:/ www.mdpi.com/2306-738 1/8/3/37/s1: Table S1: Comparison Species and GenBank Accession Numbers, Table S2: BLASTn results and sex of infected ticks, Data S3: Raw sequence data of all Rickettsia samples amplified from ticks collected on companion animals. Figure S1: Phylogenetic tree of all Rickettsia species identified and their associated tick identification numbers.

Author Contributions: The first author of this paper, H.S., contributed to the study conceptualization and design, lab work, interpretation of results, and preparation of the manuscript. D.V.L.R. contributed to the study conceptualization and design, interpretation of results, and preparation of the manuscript. All authors have read and agreed to the published version of the manuscript.

Funding: This research was funded by a Berry College Kirbo Undergraduate Scholar Grant awarded to Hannah Stanley.

Institutional Review Board Statement: Not applicable.

Informed Consent Statement: Not applicable.

Data Availability Statement: All data generated in this study can be found in Supplemental Materials.

Acknowledgments: The authors thank the Murrayville Veterinary Clinic, Susan Hanson, and Adrian Hanson-Lopez for the generous donation of the ticks used in this study. Additionally, the authors would like to acknowledge the hard work of Austin Fowler, Ethan Hollis, and Ethan Hart in the completion of this study.

Conflicts of Interest: The authors declare no conflict of interest.

\section{References}

1. Kilpatrick, A.M.; Dobson, A.D.M.; Levi, T.; Salkeld, D.J.; Swei, A.; Ginsberg, H.S.; Kjemtrup, A.; Padgett, K.A.; Jensen, P.M.; Fish, D.; et al. Lyme Disease Ecology in a Changing World: Consensus, Uncertainty and Critical Gaps for Improving Control. Philos. Trans. R. Soc. Lond. B Biol. Sci. 2017, 372. [CrossRef]

2. Sonenshine, D.E. Range Expansion of Tick Disease Vectors in North America: Implications for Spread of Tick-Borne Disease. Int. J. Environ. Res. Public Health 2018, 15, 478. [CrossRef] 
3. Bouchard, C.; Dibernardo, A.; Koffi, J.; Wood, H.; Leighton, P.A.; Lindsay, L.R. N Increased Risk of Tick-Borne Diseases with Climate and Environmental Changes. Can. Commun. Dis. Rep. 2019, 45, 83-89. [CrossRef] [PubMed]

4. McQuiston, J.H.; Paddock, C.D.; Holman, R.C.; Childs, J.E. The Human Ehrlichioses in the United States. Emerg. Infect. Dis. 1999, 5, 635-642. [CrossRef]

5. Gleim, E.R.; Garrison, L.E.; Vello, M.S.; Savage, M.Y.; Lopez, G.; Berghaus, R.D.; Yabsley, M.J. Factors Associated with Tick Bites and Pathogen Prevalence in Ticks Parasitizing Humans in Georgia, USA. Parasites Vectors 2016, 9, 125. [CrossRef]

6. Cohen, S.B.; Yabsley, M.J.; Garrison, L.E.; Freye, J.D.; Dunlap, B.G.; Dunn, J.R.; Mead, D.G.; Jones, T.F.; Moncayo, A.C. Rickettsia Parkeri in Amblyomma Americanum Ticks, Tennessee and Georgia, USA. Emerg. Infect. Dis. 2009, 15, 1471-1473. [CrossRef] [PubMed]

7. Moncayo, A.C.; Cohen, S.B.; Fritzen, C.M.; Huang, E.; Yabsley, M.J.; Freye, J.D.; Dunlap, B.G.; Huang, J.; Mead, D.G.; Jones, T.F.; et al. Absence of Rickettsia Rickettsii and Occurrence of Other Spotted Fever Group Rickettsiae in Ticks from Tennessee. Am. J. Trop. Med. Hyg. 2010, 83, 653-657. [CrossRef] [PubMed]

8. $\quad$ Fritzen, C.M.; Huang, J.; Westby, K.; Freye, J.D.; Dunlap, B.; Yabsley, M.J.; Schardein, M.; Dunn, J.R.; Jones, T.F.; Moncayo, A.C. Infection Prevalences of Common Tick-Borne Pathogens in Adult Lone Star Ticks (Amblyomma Americanum) and American Dog Ticks (Dermacentor Variabilis) in Kentucky. TAm. J. Trop. Med. Hyg. 2011, 85, 718-723. [CrossRef]

9. Sayler, K.A.; Loftis, A.D.; Beatty, S.K.; Boyce, C.L.; Garrison, E.; Clemons, B.; Cunningham, M.; Alleman, A.R.; Barbet, A.F. Prevalence of Tick-Borne Pathogens in Host-Seeking Amblyomma Americanum (Acari: Ixodidae) and Odocoileus Virginianus (Artiodactyla: Cervidae) in Florida. J. Med. Entomol. 2016, 53, 949-956. [CrossRef] [PubMed]

10. Allerdice, M.E.J.; Hecht, J.A.; Lash, R.R.; Karpathy, S.E.; Paddock, C.D. Rickettsia Parkeri and "Candidatus Rickettsia Andeanae" in Amblyomma Maculatum (Acari: Ixodidae) Collected from the Atlanta Metropolitan Area, Georgia, United States. Ticks Tick Borne Dis. 2019, 10, 1066-1069. [CrossRef] [PubMed]

11. Weinert, L.A.; Werren, J.H.; Aebi, A.; Stone, G.N.; Jiggins, F.M. Evolution and Diversity of Rickettsia Bacteria. BMC Biol. 2009, 7, 6. [CrossRef]

12. Paddock, C.D.; Sumner, J.W.; Comer, J.A.; Zaki, S.R.; Goldsmith, C.S.; Goddard, J.; McLellan, S.L.F.; Tamminga, C.L.; Ohl, C.A. Rickettsia Parkeri: A Newly Recognized Cause of Spotted Fever Rickettsiosis in the United States. Clin. Infect. Dis. 2004, 38, 805-811. [CrossRef] [PubMed]

13. Dantas-Torres, F. Rocky Mountain Spotted Fever. Lancet Infect. Dis. 2007, 7, 724-732. [CrossRef]

14. Parola, P. Rickettsia Felis: From a Rare Disease in the USA to a Common Cause of Fever in Sub-Saharan Africa. Clin. Microbiol. Infect. 2011, 17, 996-1000. [CrossRef]

15. Brown, L.D.; Macaluso, K.R. Rickettsia Felis, an Emerging Flea-Borne Rickettsiosis. Curr. Trop. Med. Rep. 2016, 3, 27-39. [CrossRef]

16. Labruna, M.B.; Whitworth, T.; Bouyer, D.H.; McBride, J.; Camargo, L.M.A.; Camargo, E.P.; Popov, V.; Walker, D.H. Rickettsia Bellii and Rickettsia Amblyommii in Amblyomma Ticks from the State of Rondônia, Western Amazon, Brazil. J. Med Entomol. 2004, 41, 1073-1081. [CrossRef] [PubMed]

17. Spach, D.H.; Liles, W.C.; Campbell, G.L.; Quick, R.E.; Anderson, D.E.; Fritsche, T.R. Tick-Borne Diseases in the United States. N. Engl. J. Med. 1993, 329, 936-947. [CrossRef] [PubMed]

18. Shaw, S.E.; Day, M.J.; Birtles, R.J.; Breitschwerdt, E.B. Tick-Borne Infectious Diseases of Dogs. Trends Parasitol. 2001, 17, 74-80. [CrossRef]

19. Fritz, C.L.; Kriner, P.; Garcia, D.; Padgett, K.A.; Espinosa, A.; Chase, R.; Hu, R.; Messenger, S.L. Tick Infestation and Spotted-Fever Group Rickettsia in Shelter Dogs, California, 2009. Zoonoses Public Health 2012, 59, 4-7. [CrossRef]

20. Greene, C.E.; Burgdorfer, W.; Cavagnolo, R.; Philip, R.N.; Peacock, M.G. Rocky Mountain Spotted Fever in Dogs and Its Differentiation from Canine Ehrlichiosis. J. Am. Vet. Med. Assoc. 1985, 186, 465-472.

21. Keirans, J.E.; Litwak, T.R. Pictorial Key to the Adults of Hard Ticks, Family Ixodidae (Ixodida: Ixodoidea), East of the Mississippi River. J. Med. Entomol. 1989, 26, 435-448. [CrossRef]

22. Blair, P.J.; Jiang, J.; Schoeler, G.B.; Moron, C.; Anaya, E.; Cespedes, M.; Cruz, C.; Felices, V.; Guevara, C.; Mendoza, L.; et al. Characterization of Spotted Fever Group Rickettsiae in Flea and Tick Specimens from Northern Peru. J. Clin. Microbiol. 2004, 42, 4961-4967. [CrossRef]

23. Larkin, M.A.; Blackshields, G.; Brown, N.P.; Chenna, R.; McGettigan, P.A.; McWilliam, H.; Valentin, F.; Wallace, I.M.; Wilm, A.; Lopez, R.; et al. Clustal W and Clustal X Version 2.0. Bioinformatics 2007, 23, 2947-2948. [CrossRef]

24. Guindon, S.; Dufayard, J.-F.; Lefort, V.; Anisimova, M.; Hordijk, W.; Gascuel, O. New Algorithms and Methods to Estimate Maximum-Likelihood Phylogenies: Assessing the Performance of PhyML 3.0. Syst. Biol. 2010, 59, 307-321. [CrossRef]

25. Theis, J.H. Mechanical Removal of Rhipicephalus Sanguineus from the Dog. J. Am. Vet. Med. Assoc. 1968, 153, 433-437. [PubMed]

26. Rhodes, A.R.; Norment, B.R. Hosts of Rhipicephalus Sanguineus (Acari: Ixodidae) in Northern Mississippi, USA. J. Med. Entomol. 1979, 16, 488-492. [CrossRef] [PubMed]

27. Ogden, N.H.; Pang, G.; Ginsberg, H.S.; Hickling, G.J.; Burke, R.L.; Beati, L.; Tsao, J.I. Evidence for Geographic Variation in Life-Cycle Processes Affecting Phenology of the Lyme Disease Vector Ixodes Scapularis (Acari: Ixodidae) in the United States. J. Med. Entomol. 2018, 55, 1386-1401. [CrossRef] [PubMed]

28. Hii, S.F.; Kopp, S.R.; Abdad, M.Y.; Thompson, M.F.; O'Leary, C.A.; Rees, R.L.; Traub, R.J. Molecular Evidence Supports the Role of Dogs as Potential Reservoirs for Rickettsia Felis. Vector Borne Zoonotic Dis. 2011, 11, 1007-1012. [CrossRef] 
29. Duh, D.; Punda-Polić, V.; Trilar, T.; Petrovec, M.; Bradarić, N.; Avsic-Zupanc, T. Molecular Identification of Rickettsia Felis-like Bacteria in Haemaphysalis Sulcata Ticks Collected from Domestic Animals in Southern Croatia. Ann. N. Y. Acad. Sci. 2006, 1078, 347-351. [CrossRef]

30. Ishikura, M.; Ando, S.; Shinagawa, Y.; Matsuura, K.; Hasegawa, S.; Nakayama, T.; Fujita, H.; Watanabe, M. Phylogenetic Analysis of Spotted Fever Group Rickettsiae Based on GltA, 17-KDa, and ROmpA Genes Amplified by Nested PCR from Ticks in Japan. Microbiol. Immunol. 2003, 47, 823-832. [CrossRef]

31. Oliveira, K.A.; Oliveira, L.S.; Dias, C.C.A.; Silva, A.; Almeida, M.R.; Almada, G.; Bouyer, D.H.; Galvão, M.A.M.; Mafra, C. Molecular Identification of Rickettsia Felis in Ticks and Fleas from an Endemic Area for Brazilian Spotted Fever. Mem. Inst. Oswaldo Cruz 2008, 103, 191-194. [CrossRef]

32. Jiang, J.; Stromdahl, E.Y.; Richards, A.L. Detection of Rickettsia Parkeri and Candidatus Rickettsia Andeanae in Amblyomma Maculatum Gulf Coast Ticks Collected from Humans in the United States. Vector Borne Zoonotic Dis. 2012, 12, 175-182. [CrossRef]

33. Pornwiroon, W.; Pourciau, S.S.; Foil, L.D.; Macaluso, K.R. Rickettsia Felis from Cat Fleas: Isolation and Culture in a Tick-Derived Cell Line. Appl. Environ. Microbiol. 2006, 72, 5589-5595. [CrossRef]

34. Apperson, C.S.; Engber, B.; Nicholson, W.L.; Mead, D.G.; Engel, J.; Yabsley, M.J.; Dail, K.; Johnson, J.; Watson, D.W. Tick-Borne Diseases in North Carolina: Is "Rickettsia Amblyommii" a Possible Cause of Rickettsiosis Reported as Rocky Mountain Spotted Fever? Vector Borne Zoonotic Dis. 2008, 8, 597-606. [CrossRef] [PubMed]

35. Delisle, J.; Mendell, N.L.; Stull-Lane, A.; Bloch, K.C.; Bouyer, D.H.; Moncayo, A.C. Human Infections by Multiple Spotted Fever Group Rickettsiae in Tennessee. Am. J. Trop. Med. Hyg. 2016, 94, 1212-1217. [CrossRef]

36. Karpathy, S.E.; Slater, K.S.; Goldsmith, C.S.; Nicholson, W.L.; Paddock, C.D. Rickettsia Amblyommatis Sp. Nov., a Spotted Fever Group Rickettsia Associated with Multiple Species of Amblyomma Ticks in North, Central and South America. Int. J. Syst. Evol. Microbiol. 2016, 66, 5236-5243. [CrossRef] [PubMed]

37. Vaughn, M.F.; Delisle, J.; Johnson, J.; Daves, G.; Williams, C.; Reber, J.; Mendell, N.L.; Bouyer, D.H.; Nicholson, W.L.; Moncayo, A.C.; et al. Seroepidemiologic Study of Human Infections with Spotted Fever Group Rickettsiae in North Carolina. J. Clin. Microbiol. 2014, 52, 3960-3966. [CrossRef] [PubMed]

38. Barrett, A.; Little, S.E.; Shaw, E. "Rickettsia Amblyommii" and R. Montanensis Infection in Dogs Following Natural Exposure to Ticks. Vector Borne Zoonotic Dis. 2013, 14, 20-25. [CrossRef]

39. Nicholson, W.L.; Allen, K.E.; McQuiston, J.H.; Breitschwerdt, E.B.; Little, S.E. The Increasing Recognition of Rickettsial Pathogens in Dogs and People. Trends Parasitol. 2010, 26, 205-212. [CrossRef] [PubMed]

40. Breitschwerdt, E.B.; Walker, D.H.; Levy, M.G.; Burgdorfer, W.; Corbett, W.T.; Hurlbert, S.A.; Stebbins, M.E.; Curtis, B.C.; Allen, D.A. Clinical, Hematologic, and Humoral Immune Response in Female Dogs Inoculated with Rickettsia Rickettsii and Rickettsia Montana. Am. J. Vet. Res. 1988, 49, 70-76. [PubMed]

41. McQuiston, J.H.; Zemtsova, G.; Perniciaro, J.; Hutson, M.; Singleton, J.; Nicholson, W.L.; Levin, M.L. Afebrile Spotted Fever Group Rickettsia Infection after a Bite from a Dermacentor Variabilis Tick Infected with Rickettsia Montanensis. Vector Borne Zoonotic Dis. 2012, 12, 1059-1061. [CrossRef] [PubMed] 